\title{
Erratum: Axiomatic approach to contextuality and nonlocality [Phys. Rev. A 92, 032104 (2015)]
}

\author{
Karol Horodecki, Andrzej Grudka, Pankaj Joshi, Waldemar Kłobus, and Justyna Łodyga
}

(Received 26 February 2019; published 13 March 2019)

DOI: 10.1103/PhysRevA.99.039901

In our previous paper unfortunate notation had been chosen, which could lead to misunderstandings. In this Erratum we wish to clarify it in several respects, regarding changes in Secs. III and IV.

In Eq. (20) (and thereinafter) of the original paper we write $P\left(\lambda_{r} \mid c\right)$ as a joint probability distribution for all the observables from the set $\mathcal{M} \backslash c$, where $\lambda_{r}$ is the corresponding set of measurement outcomes. Instead, it should read $P\left(\lambda_{r} \mid \lambda_{c}\right)$ as a joint probability distribution for all the observables from the set $\mathcal{M} \backslash c$ with $\lambda_{r}$ as the corresponding set of measurement outcomes, conditioned on the outcomes of $c$ given by $\lambda_{c}$. The same notation for $P\left(\lambda_{r} \mid \lambda_{c}\right)$ should be used below Eq. (21), in Eqs. (30)-(32), and below Eq. (32).

Additionally, the following clarification concerning another point ought to be addressed. Below Eq. (44) there is " $d=$ $\min \left(\prod_{i=1}^{k} a_{i},\left|E_{G}\right|\right)$, where $\prod_{i=1}^{k} a_{i}$ and $\left|E_{G}\right|$ are the dimensions of the two subsystems of the box $B$, respectively." It was referring to two marginals of the distribution $\sum_{c} p(c)|c\rangle\langle c| \otimes \mathcal{A}_{B}(c)$ and should read " $d=\min \left(\prod_{i=1}^{|\mathcal{M}|} a_{i},|\mathcal{C}|\right)$, where $|\mathcal{M}|$ is the cardinality of $\mathcal{M}, a_{i}$ is the number of outcomes of the $i$ th observable from $\mathcal{M}$, and $|\mathcal{C}|$ is the number of contexts within $\mathcal{M}$."

We wish to stress that these changes do not in any way affect the validity of the theorems and other contents presented in the original paper. These changes concern only Secs. III (regarding notation) and IV (regarding its consequence) and affect only the form, not the substance, of the main result of these sections, which is the asymptotic continuity of the relative entropy of contextuality. 